\title{
Migration Using a Transversely Isotropic Medium with Symmetry Normal to the Reflector Dip
}

\author{
Tariq Alkhalifah ${ }^{1}$ and Paul Sava ${ }^{2}$ \\ ${ }^{1}$ King Abdullah University of Science and Technology, Thuwal, Saudi Arabia \\ ${ }^{2}$ Center for Wave Phenomena, Colorado School of Mines, Golden, CO 80401, USA \\ Correspondence should be addressed to Tariq Alkhalifah, tariq.alkhalifah@kaust.edu.sa
}

Received 14 December 2010; Accepted 10 March 2011

Academic Editor: Martin Tygel

Copyright ( $\odot 2011$ T. Alkhalifah and P. Sava. This is an open access article distributed under the Creative Commons Attribution License, which permits unrestricted use, distribution, and reproduction in any medium, provided the original work is properly cited.

\begin{abstract}
A transversely isotropic (TI) model in which the tilt is constrained to be normal to the dip (DTI model) allows for simplifications in the imaging and velocity model building efforts as compared to a general TI (TTI) model. Although this model cannot be represented physically in all situations, for example, in the case of conflicting dips, it handles arbitrary reflector orientations under the assumption of symmetry axis normal to the dip. Using this assumption, we obtain efficient downward continuation algorithms compared to the general TTI ones, by utilizing the reflection features of such a model. Phase-shift migration can be easily extended to approximately handle lateral inhomogeneity using, for example, the split-step approach. This is possible because, unlike the general TTI case, the DTI model reduces to VTI for zero dip. These features enable a process in which we can extract velocity information by including tools that expose inaccuracies in the velocity model in the downward continuation process. We test this model on synthetic data corresponding to a general TTI medium and show its resilience.
\end{abstract}

\section{Introduction}

Migration velocity analysis (MVA), despite the many developments in recent years, is still a challenging process especially in complex media. MVA is even more of a challenge in anisotropic media in which the medium is described by several parameters, all of which can change as a function of position. Anisotropy introduces flexibility to the model to better simulate the Earth subsurface, but it also introduces a null space to the parameter estimation process or MVA. As a result, we need to use anisotropy to allow for more freedom up to the point where data seize to influence the model, or even part of it. This anisotropy null-space tradeoff has recently guided us to using a transversely isotropic (TI) medium with a tilted axis of symmetry (TTI). To avoid the null space, such tilt is assumed to be in the direction of the dip [1, 2]. In fact, Audebert et al. [2] referred to this medium as structurally conformed transversely isotropic (STI), in which parameter description becomes simpler by fixing the symmetry axis to be normal to the structure. In their formulation, the symmetry axis field is measured from the image (a representation of the structure) and used along with regular TTI equations to implement migration and velocity analysis.

In transversely isotropic with vertical symmetry axis (VTI) media, the acoustic problem can be described by three parameters [3]: the velocity in the symmetry direction, the normal-moveout (NMO) velocity measured with respect to the symmetry direction (related to the second derivative of the phase velocity with respect to phase angle at the symmetry direction), and an anisotropy parameter that relates the NMO velocity to the velocity in the direction normal to the symmetry axis, usually labeled $\eta$. For TI media with a tilt in the axis of symmetry, two angles, that describe the tilt in $3 \mathrm{D}$, are also needed to fully characterize acoustic wave propagation.

Alkhalifah and Sava [4] introduce the concept of using the assumption that symmetry axis is normal to the reflector dip as a constrain to develop simplified and explicit representations for moveout in extended images, for angle gather mapping, and for migration. They refer to the model 
as dip-constrained TI (DTI). In this paper, we use the explicit formulations provided by the DTI model to perform downward continuation and discuss its potential for TTI parameter estimation. In this context, many of the familiar tools developed for the isotropic case apply with little or no modifications. We utilize this to build a framework for imaging and velocity model building for DTI models and illustrate the method with synthetic data.

\section{Downward Continuation}

Downward continuation, with the double-square equation in the DTI framework, utilizes the equal incidence and reflection angles imposed by the constraint. As shown by Alkhalifah and Sava [4], the downward continuation process includes two steps: determining the offset wavenumber corresponding to a particular reflection angle and then using the offset wavenumber to determine the required phase shift based on the double-square-root (DSR) equation. The two steps are given by explicit equations that can be implemented using the algorithm suggested by Alkhalifah and Sava [4].

Considering that angle gather extraction is a localized process relying on the plane wave behavior around the scattering point, it is applicable, within the limits of high frequencies compared to medium variations, to complex media. To allow the DSR-based downward continuation to honor lateral inhomogeneity at least approximately, we can utilize the phase-shift-plus-interpolation concept [5]. In this case, we downward continue multiple times for various symmetry-direction anisotropy parameters and then interpolate the wavefields using the lateral velocity information in space. However, the split-step approach of Stoffa et al. [6] lends itself better to this approach as we maintain reflection angle information in the space domain needed for a good zero-dip velocity, $v(x, y, z)$, correction.

Wavefield reconstruction for multioffset migration based on the one-way wave equation under the survey-sinking framework [7] is implemented by recursive phase shift of prestack wavefields

$$
u_{z+\Delta z}(\mathbf{m}, \mathbf{h})=e^{-i k_{z} \Delta z} u_{z}(\mathbf{m}, \mathbf{h}),
$$

followed by extraction of the image at time $t=0$. Here, $\mathbf{m}$ and $\mathbf{h}$ represent the midpoint and half offset coordinates. In (1), $u_{z}(\mathbf{m}, \mathbf{h})$ represents the acoustic wavefield for a given frequency $\omega$ at all midpoint positions $\mathbf{m}$ and half offsets $\mathbf{h}$ at depth $z$, and $u_{z+\Delta z}(\mathbf{m}, \mathbf{h})$ represents the same wavefield extrapolated to depth $z+\Delta z$. The phase shift in laterally homogeneous media is given by the depth wavenumber $k_{z}$ which is defined by the DSR formula. In the case of the $2 \mathrm{D}$ DTI model, the dispersion relation is given by the following formula [4]:

$$
\begin{aligned}
k_{z} & =k_{z s}+k_{z r} \\
& =\sqrt{\frac{\omega^{2}}{v_{p}^{2}(\theta)}-\left(k_{m}-k_{h}\right)^{2}}+\sqrt{\frac{\omega^{2}}{v_{p}^{2}(\theta)}-\left(k_{m}+k_{h}\right)^{2}},
\end{aligned}
$$

where $\omega$ is the angular frequency, $k_{m}$ is the midpoint wavenumber, $v_{p}$ is the phase velocity as a function of the reflection angle (half of the scattering angle for DTI), $\theta$, and $k_{z s}$ and $k_{z r}$ are the source and receiver vertical wavenumbers, respectively. The offset wavenumber, $k_{h}$, is evaluated using the following formula:

$$
k_{h}^{2}(\theta)=\left(\frac{2 \omega \sin \theta}{v_{p}(\theta)}\right)^{2}-k_{m}^{2} \tan ^{2} \theta .
$$

To implement such a phase shift downward continuation, we loop, per depth step, over $\omega, k_{m}$, and most importantly $\theta$. The phase velocity used in (2) can be regarded as a reference velocity in laterally inhomogeneous media corresponding to anisotropy parameters, for example, from the center of the model. For a split-step implementation, we apply a space domain correction corresponding to zero dip. Thus, the phase correction in the space domain has the following form:

$$
k_{z c}=2 \sqrt{\frac{\omega^{2}}{v_{p}^{2}(x, \theta)}-k_{h}^{2}}-2 \sqrt{\frac{\omega^{2}}{v_{p}^{2}(\theta)}-k_{h}^{2}},
$$

where $v_{p}(x, \theta)$ is the laterally varying phase velocity corresponding to anisotropic parameters at their space location. The offset wave number is given now by a simpler equation

$$
k_{h}(x, \theta)=\frac{2 \omega \sin \theta}{v_{p}(x, \theta)} .
$$

For completeness, the laterally varying phase velocity for TI media is given as

$$
\begin{aligned}
v_{p}^{2}(x, \theta)= & \frac{1}{2}\left(a \sin ^{2} \theta+b \cos ^{2} \theta\right) \\
& +\frac{1}{4} \sqrt{4 a^{2} \sin ^{4} \theta+2 a b \sin ^{2}(2 \theta)+4 b^{2} \cos ^{4} \theta},
\end{aligned}
$$

where $a=v^{2}(x)(1+2 \eta(x)), b=v_{T}^{2}(x), v_{T}$ is the velocity in the symmetry direction, $v$ is the NMO velocity with respect to the symmetry axis, and $\eta$ is the anisotropy parameter relating the NMO velocity to the velocity normal to the symmetry axis. The angle $\theta$ in (6) is measured from the symmetry axis. Here, we are relying on the zero dip for the lateral correction, though the model is DTI, which implies a VTI model for this dip.

\section{Velocity Analysis}

Having an analytical representation for the migration operator allows us to develop migration analysis tools. The dependency of the migration operator on medium parameters is at the heart of such developments. Using the Sava and Vlad [8] approach to develop the velocity analysis operator in 3D, we can assume the separation of the extrapolation slowness $s(\mathbf{m})=1 / v_{p}^{2}(\theta)$ into a background component $s_{0}(\mathbf{m})$ and an unknown perturbation component $\Delta s(\mathbf{m})$. Then, we can construct a wavefield perturbation $\Delta u(\mathbf{m}, \mathbf{h})$ at depth $z$ and frequency $\omega$ related linearly to the slowness perturbation $\Delta s(\mathbf{m})$. Linearizing the depth wavenumber given by the DSR equation (2) relative to the background slowness $s_{0}(\mathbf{m})$, we obtain

$$
k_{z} \approx k_{z 0}+\left.\frac{d k_{z_{s}}}{d s}\right|_{s_{0}} \Delta s(\mathbf{m}-\mathbf{h})+\left.\frac{d k_{z r}}{d s}\right|_{s_{0}} \Delta s(\mathbf{m}+\mathbf{h}),
$$


where the depth wavenumber in the background medium is

$$
\begin{aligned}
& k_{z 0}= \sqrt{\left[\omega s_{0}(\mathbf{m}-\mathbf{h})\right]^{2}-\left|\frac{\mathbf{k}_{\mathbf{x}}-\mathbf{k}_{\mathbf{h}}}{2}\right|^{2}} \\
&+\sqrt{\left[\omega s_{0}(\mathbf{m}+\mathbf{h})\right]^{2}-\left|\frac{\mathbf{k}_{\mathbf{x}}+\mathbf{k}_{\mathbf{h}}}{2}\right|^{2}},
\end{aligned}
$$

and $\mathbf{k}_{\mathbf{x}}$ and $\mathbf{k}_{\mathbf{h}}$ are the wavenumber vectors for the midpoint and half offset, respectively, in 3D. Here, $s_{0}(x, \theta)$ represents the spatially variable background slowness at depth level $z$. Using the wavenumber linearization given by (7), we can reconstruct the acoustic wavefields in the background model using a phase-shift operation

$$
u_{z+\Delta z}(\mathbf{m}, \mathbf{h})=e^{-i k_{z 0} \Delta z} u_{z}(\mathbf{m}, \mathbf{h}) .
$$

We can represent wavefield extrapolation using a generic solution to the one-way wave equation. This indicates that the wavefield $u_{z+\Delta z}(\mathbf{m}, \mathbf{h})$ is reconstructed from the wavefield $u_{z}(\mathbf{m}, \mathbf{h})$ using the background slowness $s_{0}(\mathbf{m})$. This operation is repeated independently for all frequencies $\omega$.

However, our desire is to relate the wavefield perturbation directly to medium perturbation, not just the phase velocity. For the DTI model, $s(\mathbf{m})$ is dependent on the TI model parameters, which for the acoustic case are $v_{T}, v$, and $\eta$. Thus, (7) becomes

$$
\begin{aligned}
k_{z} \approx & k_{z 0}+\left.\frac{d k_{z s}}{d s} \frac{\partial s}{\partial v_{T}}\right|_{s_{0}} \Delta v_{T}(\mathbf{m}-\mathbf{h}) \\
& +\left.\frac{d k_{z s}}{d s} \frac{\partial s}{\partial v}\right|_{s_{0}} \Delta v(\mathbf{m}-\mathbf{h}) \\
& +\left.\frac{d k_{z s}}{d s} \frac{\partial s}{\partial \eta}\right|_{s_{0}} \Delta \eta(\mathbf{m}-\mathbf{h}) \\
& +\left.\frac{d k_{z r}}{d s} \frac{\partial s}{\partial v_{T}}\right|_{s_{0}} \Delta v_{T}(\mathbf{m}+\mathbf{h}) \\
& +\left.\frac{d k_{z r}}{d s} \frac{\partial s}{\partial v}\right|_{s_{0}} \Delta v(\mathbf{m}+\mathbf{h}) \\
& +\left.\frac{d k_{z r}}{d s} \frac{\partial s}{\partial \eta}\right|_{s_{0}} \Delta \eta(\mathbf{m}+\mathbf{h}),
\end{aligned}
$$

where $s(\mathbf{m})=1 / v_{p}^{2}(\theta)$ is given by (6), and thus, the partial derivatives in (10) can be obtained analytically. We can focus on perturbation in one parameter (i.e, $\eta$ ) and thus end up with a one-to-one linearized relation between the image field and that parameter. For a detailed implementation of such an approach, we refer to [8].

\section{Dip-Constrained TTI: Not a Physical Model}

One question that arises is how does the DTI constraint be imposed on a model? Specifically, what happens when we have conflicting dips? For the equations developed here and especially those of Alkhalifah and Sava [4], based on a plane

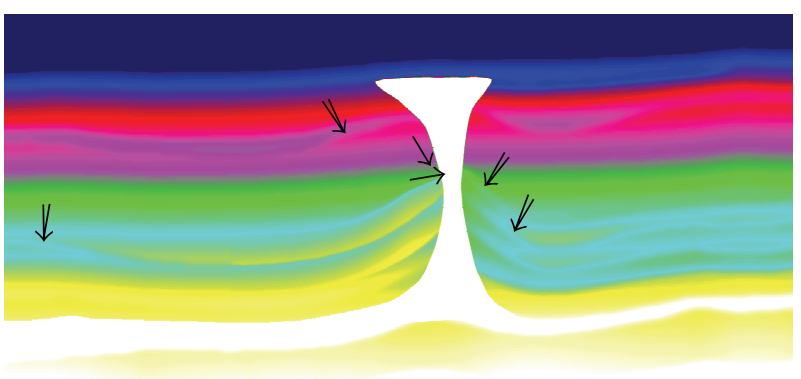

FIGURE 1: Part of BP anisotropic velocity model that contains a salt body. The abrupt change in velocity magnitude can be interpreted as reflections, and the arrows point to examples of the possible directions of TI symmetry tilt to accommodate a DTI model.

wave representation, the DTI constraint is explicitly handled in the formulations. This implies that even for conflicting dips at some position in space, the symmetry axis is always normal to the reflector dip, as if the dips were handled in separate planes. This can not be represented in the physical space, and thus, it is a process. If conflicting dips exist, then the dip that physically adheres to the constraint is handled properly. Figure 1 shows the BP anisotropic model with arrows pointing to conflicting dips. However, the axis of symmetry is single valued, and thus, the DTI model handles the reflections that adhere to this assumption. Nevertheless, conflicting dips are truly conflicting (cross at a point) if the true velocity is used in imaging. Otherwise, their conflict is in an inaccurate position and thus do not reflect the physical behavior of the reflectors.

Thus, in the BP model shown in Figure 1, we typically use the reflections that underlay the sediments, especially those near the salt flanks, to constrain the velocity model. These sediments are assumed to be TI with the symmetry axis normal to the layering. In this case, the simplified DTI model can accurately image those sediments at a reduced effort and cost. The effort is typically spent in generating velocity models that include an additional file containing the symmetry axis direction, which is not required in the DTI model. In addition, the cost of using DTI equations is far less than TTI ones, even less than using VTI equations.

\section{The Impulse Response}

The response of imaging to a dataset that includes pulses reveals some of the features of the operator involved in the imaging process. Here, our input data to the migration includes five pulses at times $0.6,1.2,1.8,2.4$, and 3 seconds at zero offset under the common midpoint (CMP) location of $4 \mathrm{~km}$. The medium is vertically inhomogeneous with velocity increasing linearly with depth and $\eta=0$.2. Figure 2 shows the migration (response) in the prestack domain with coordinates of distance, depth, and angle gather. The three sections here and throughout represent slices in the prestack cube with the black lines representing the location of these slices relative to each other. As a result, the section on the right is simply the angle gather at location $4 \mathrm{~km}$. 
4

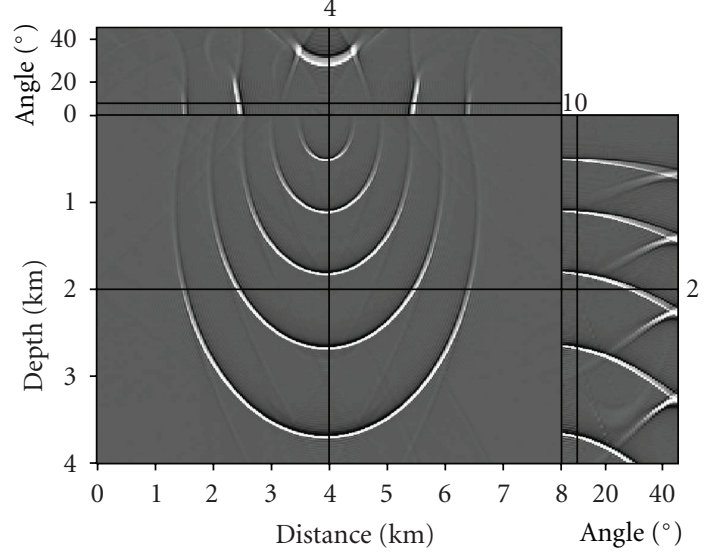

FIgURE 2: The impulse response for the prestack DTI phase-shift migration of 5 pulses at zero offset in a vertically inhomogeneous DTI model with $v(z)=1.5+0.6 z \mathrm{~km} / \mathrm{s}$ and $\eta=0.2$ and $\delta=0$. The three sections represent slices in the prestack cube with the black lines representing the location of these slices relative to each other. As a result, the section on the right is simply the angle gather at distance $4 \mathrm{~km}$.

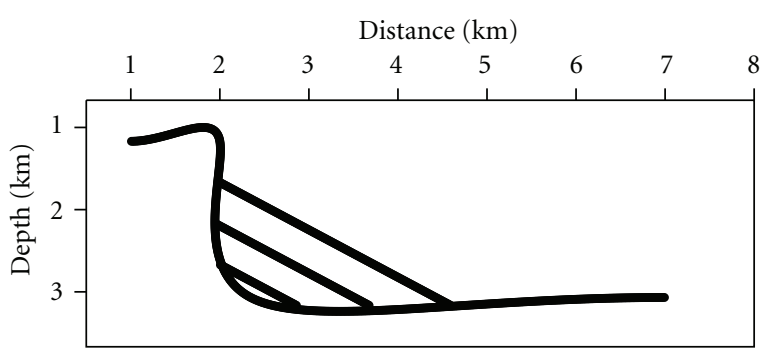

FIGURE 3: A reflector model depicting a salt flank with three parallel reflectors laying alongside the flank. The angle of symmetry is normal to the three reflectors at 30 degrees from the vertical.

As expected, the response is symmetric despite the DTI nature of the medium. Unlike the TTI case, where the symmetry axis is set to a direction, the symmetry axis here is set to be normal to the reflector, and since the response includes all dips, it will also includes all possible symmetry directions. The angle gather behavior of the impulse response completes the saddle shape of the $3 \mathrm{D}$ operator.

\section{Synthetic Example}

In the following example, we use for simplicity a vertically inhomogeneous model, although nothing in the development of processes for DTI requires that. We consider the reflector model in Figure 3, which is made up of a saltflank-like reflector in addition to three parallel reflectors depicting sediments laid over the salt flank. The TTI in this model has a symmetry axis normal to the three reflectors at $30^{\circ}$ angle from vertical. This model allows us to test the DTI concept for these three reflectors by analyzing the angle gather at $2.5 \mathrm{~km}$ location. It also allows us to observe the errors for reflectors that do not adhere to the constraint, like the salt flank and semihorizontal reflector. For a velocity

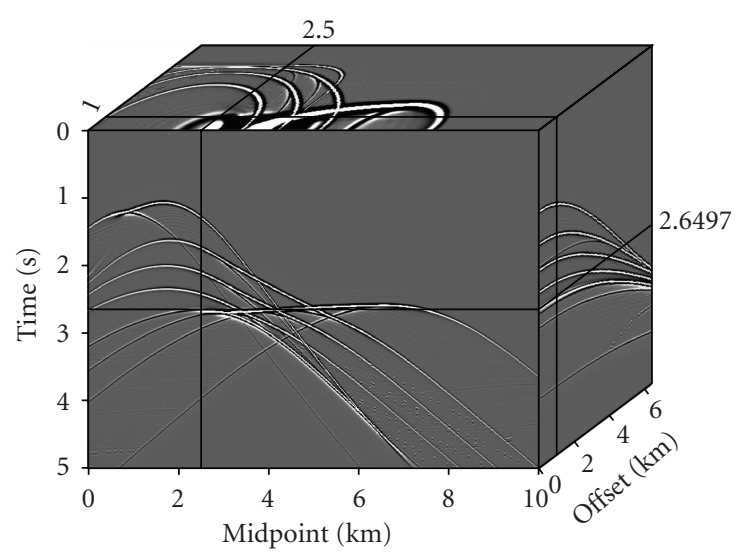

FIgUre 4: Prestack synthetic data generated using Kirchhoff modeling for the TTI model in Figure 3 with velocity (NMO and along the tilt) equals $2 \mathrm{~km} / \mathrm{s}$ and $\eta=0.2$.

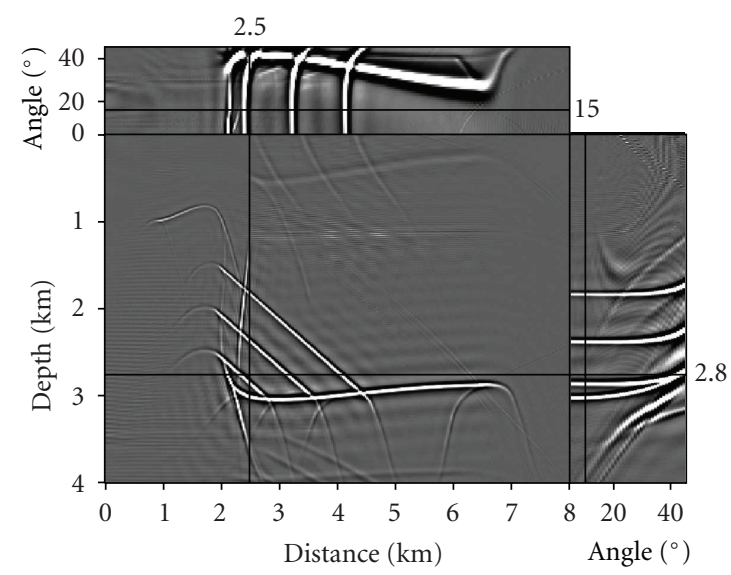

Figure 5: Migrated section after an isotropic migration with velocity of $2 \mathrm{~km} / \mathrm{s}$ of the TTI syntheticdata in Figure 4 . The angle gathers obtained using an isotropic mapping at $2.5 \mathrm{~km}$ location are displayed on the right, and the top section shows a depth slice as a function of angle gather at depth $2 \mathrm{~km}$.

of $1.5 \mathrm{~km} / \mathrm{s}$ at the surface and increasing at a gradient of $0.6 \mathrm{~s}^{-1}$, and $\eta=0.2$, we generate the prestack synthetic dataset shown in Figure 4. We use Kirchhoff modeling to obtain the synthetic data [9], and despite that the bottom reflector is semihorizontal, the clear influence of the tilt in the symmetry axis resulted in a dipping appearance on the synthetic section.

Conventional phase shift downward continuation requires that no lateral velocity variation be present. Since the synthetic model has no lateral velocity variation, we use the phase-shift approach to migrate the data. However, prior to applying the zero-lag imaging condition, we map the offset wavenumbers to angle and, thus, obtain angle gathers. Figure 5 shows the isotropically migrated section at near zero angle. It also shows on the right hand side the angle gather for isotropic angle decomposition. Clearly, the angle gather includes residuals resulting from ignoring anisotropy. They include predominantly nonhyperbolic errors associated 


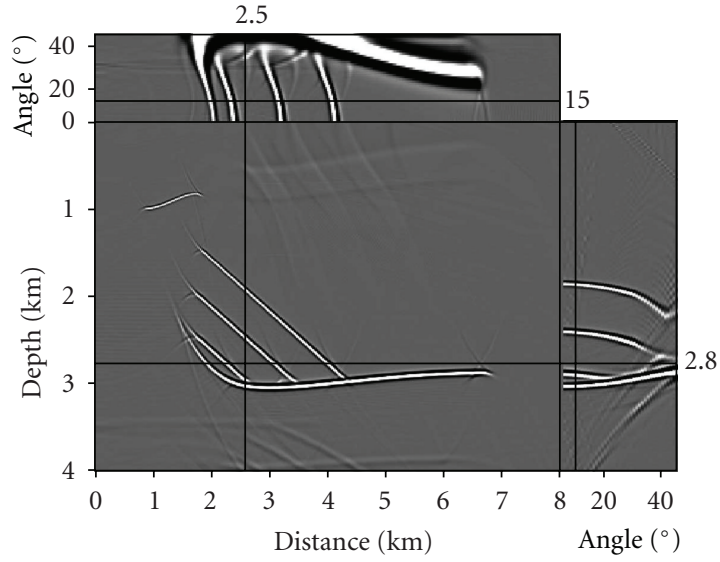

FIGURE 6: Migrated section after a VTI migration with velocity of $2 \mathrm{~km} / \mathrm{s}$ and $\eta=0.2$ of the TTI synthetic data in Figure 4. Again, the angle gathers obtained using an isotropic mapping at $8 \mathrm{~km}$ location are displayed on the right, and the top section shows a depth slice as a function of angle gather at depth $2 \mathrm{~km}$.

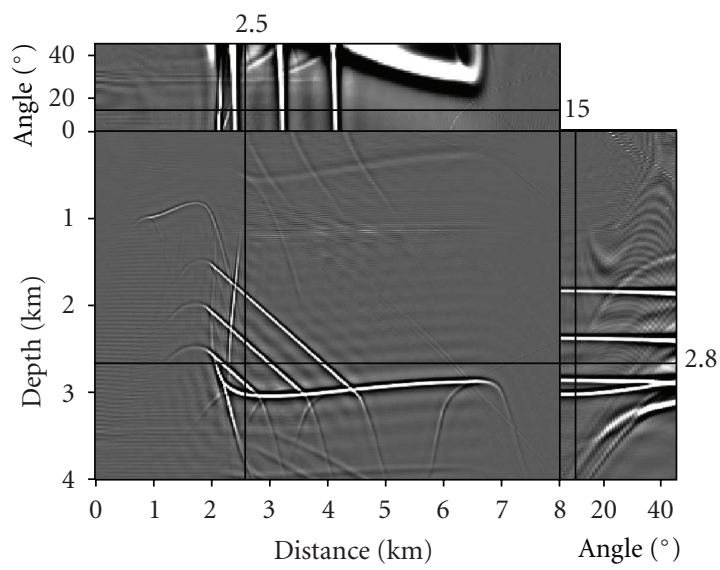

FIGURE 7: Migrated section after DTI-based migration with velocity of $2 \mathrm{~km} / \mathrm{s}$ and $\eta=0.2$ of the TTI synthetic data in Figure 4 . The angle gathers obtained, now, using the VTI mapping at $2.5 \mathrm{~km}$ location are displayed on the right. The top section shows a depth slice as a function of angle gather at depth $2 \mathrm{~km}$.

with anisotropy with some second-order errors associated with the dip [3]. The top plot in Figure 5 is a $2 \mathrm{~km}$ depth slice section and includes some residual error information spanning other angle gathers.

If we downward continue using a VTI phase-shift migration followed by an anisotropic angle gather mapping [10], we obtain the image shown in Figure 6. Both the image and the angle gathers reflect the inaccuracy of the VTI imaging for this TTI model. However, the residual moveout in the angle gathers is less than those for the isotropic case. On the other hand, the phase shift migration under the DTI assumption results in the image and angle gather shown in Figure 7. While the reflections and diffractions associated with angle not normal to the axis of symmetry show clear errors, the three parallel reflections show accurate placement and no residuals in the angle gather. This implies that the parameters used (velocity and $\eta$ ) are accurate within the DTI model that was appropriate to these reflections.

This synthetic test shows an example of the usefulness of the DTI model for analysis of key reflections. Usually, for migration velocity analysis purposes, the symmetry axis is set to be normal to the reflector dip for the reflections used in the analysis, and this is the case even for isotropic layers, which is a special case of DTI where $\eta$ and $\delta$ equal zero.

\section{Conclusions}

Constraining the symmetry axis of a transversely isotropic medium to be normal to the reflector dip (DTI) allows for explicit formulation of plane waves around the scattering point. These formulations form the basis for angle decomposition and simplified downward continuation. As a result, DTI is a convenient model for anisotropy parameter estimation in media in which such models are applicable. This model also allows us to use the general TTI assumption in a simplified form that better fits the information embedded in the recorded data. A simple synthetic example demonstrated the potential features of this model.

\section{Acknowledgment}

The authors thank KAUST and the Center of Wave Phenomena at Colorado School of Mines for their support.

\section{References}

[1] T. Alkhalifah and J. Bednar, "Building a 3-D anisotropic model: its implications to traveltime calculation and velocity analysis," in Proceedings of the 70th Annual International Meeting, Society of Exploration Geophysicists, pp. 965-968, 2000.

[2] F. S. Audebert, V. Dirks, and A. Pettenati, "TTI anisotropic depth migration: what tilt estimate should we use?" $S E G$ Technical Program Expanded Abstracts, vol. 25, no. 1, pp. 23822386, 2006.

[3] T. Alkhalifah and I. Tsvankin, "Velocity analysis for transversely isotropic media," Geophysics, vol. 60, no. 5, pp. 15501566, 1995.

[4] T. Alkhalifah and P. Sava, "A transversely isotropic medium with a tilted symmetry axis normal to the reflector," Geophysics, vol. 75, pp. A19-A24, 2010.

[5] J. Gazdag and P. Sguazzero, "Migration of seismic data by phase-shift plus interpolation," Geophysics, vol. 49, no. 2, pp. 124-131, 1984.

[6] P. L. Stoffa, J. T. Fokkema, R. M. de Luna Freire, and W. P. Kessinger, "Split-step Fourier migration," Geophysics, vol. 55, no. 4, pp. 410-421, 1990.

[7] J. F. Claerbout, Imaging the Earth's Interior, Blackwell Scientific Publications, Oxford, UK, 1985.

[8] P. Sava and I. Vlad, "Numeric implementation of waveequation migration velocity analysis operators," Geophysics, vol. 73, no. 5, pp. VE145-VE159, 2008.

[9] T. Alkhalifah, "Efficient synthetic-seismogram generation in transversely isotropic, inhomogeneous media," Geophysics, vol. 60, no. 4, pp. 1139-1150, 1995.

[10] T. Alkhalifah and S. Fomel, "Angle gathers in wave-equation imaging for vti media," SEG Technical Program Expanded Abstracts, vol. 28, pp. 2899-2903, 2009. 

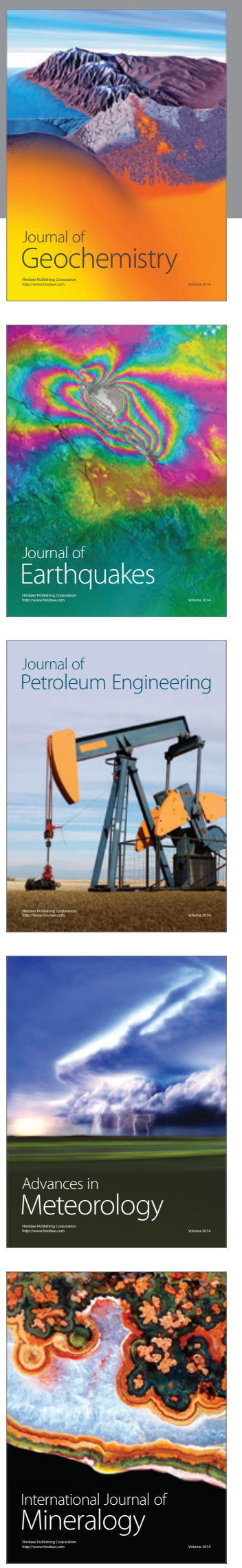
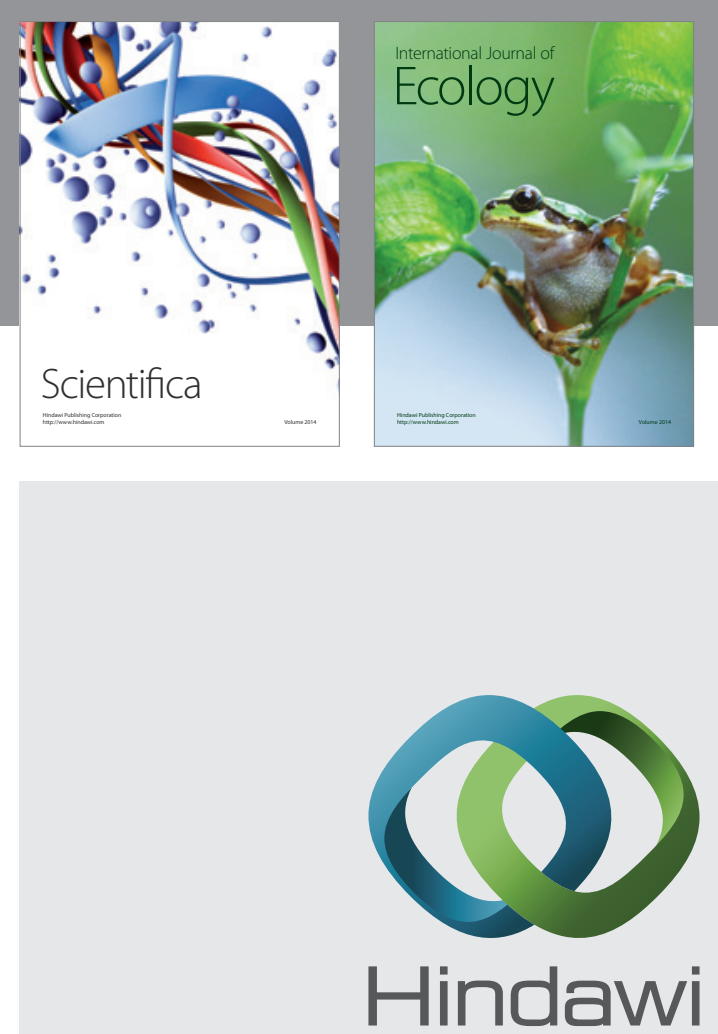

Submit your manuscripts at http://www.hindawi.com
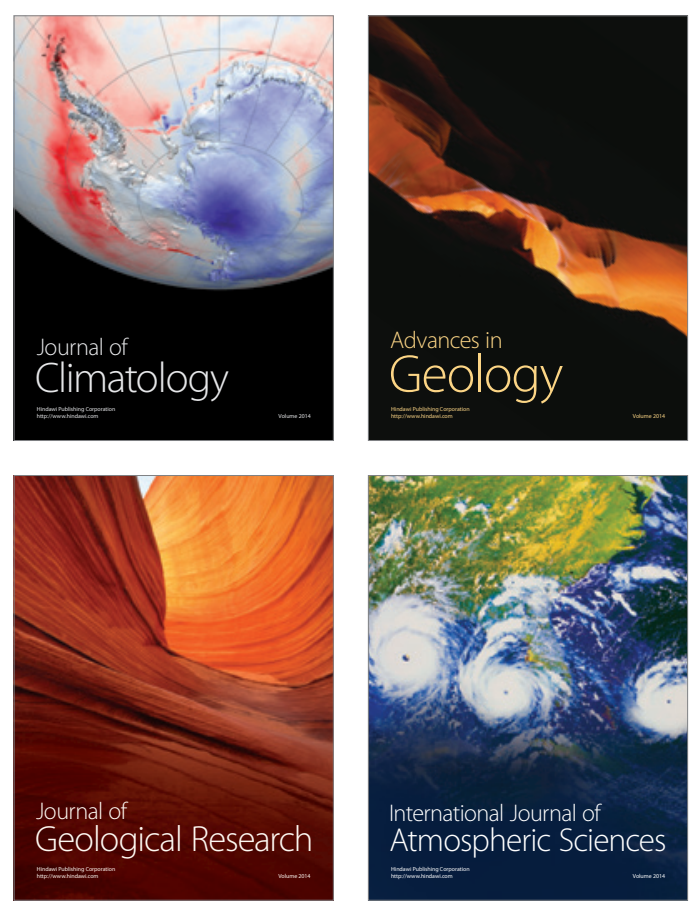
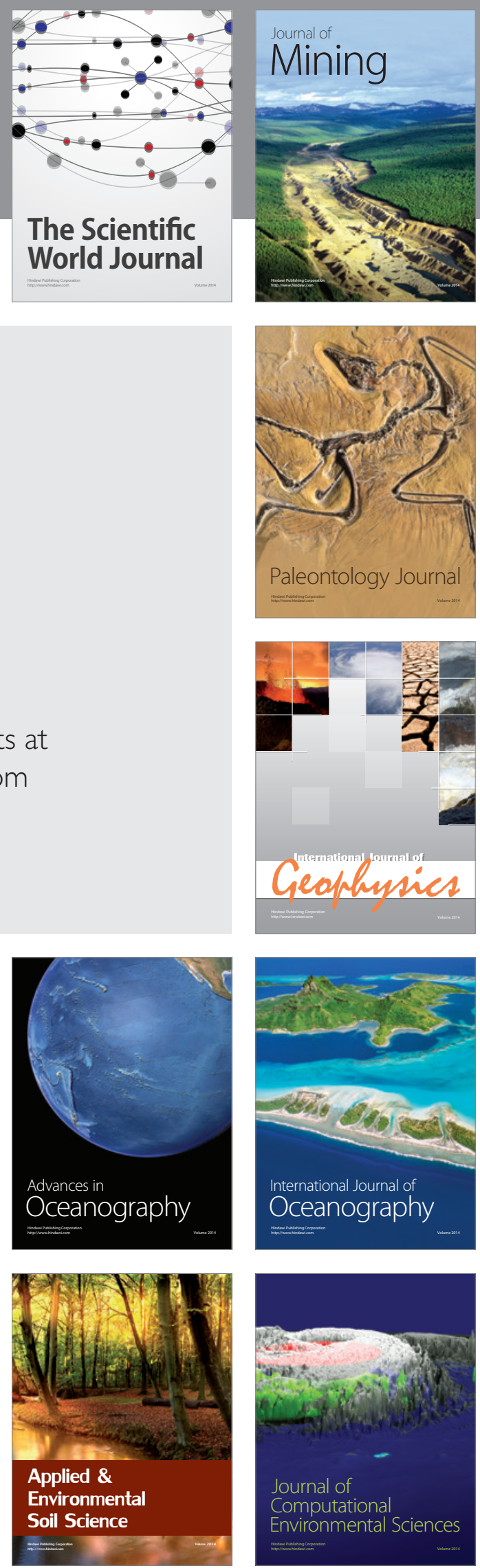